\title{
"Same journey travel network" - The reestablishment of OTA mode in tourism industry
}

\author{
Chen Hong-an, Lu Lu
}

School of Business, East China University of Science and Technology, Shanghai, China

Email address:chenha19@163.com,1065610669@qq.com

Key words: Same journey travel network, OTA mode, tourism

\begin{abstract}
Since 90s, information technology and circulation system technology develop quickly. The tourism industry, not only in the quantity, but quality of it also have undergone tremendous changes, such as the mode of tourism, the transformation about tourism business model and so on. With the demand of consumer travel is booming, same journey travel network has developed rapidly. At the same time, the rapid development of electronic information globalization also makes the rapid spread of mobile intelligent terminals. So it provides many new trading business models for the development of the tourism industry. The mode of tourism OTA has had a profound impact on the supply and consumption process of tourism industry, and the organization structure has undergone a fundamental change. The mode of tourism OTA reforms the tourism consumption concept, and may has a vast prospect.
\end{abstract}

\section{“同程旅游”一一旅游行业OTA模式的再次启航 陈洪安 鲁露}

商学院, 华东理工大学, 上海市, 中国

邮箱: chenha19@163.com, 1065610669@qq.com

中文摘要: 自上世纪九十年代以来, 信息技术, 流通系统技术不断发展, 旅游业不仅从量甚 至到质方面都发生了巨大的变化, 比如消费者的旅游行为方式, 旅游业经营模式的转变等等。 随着消费者旅游需求的不断旺盛, 同程旅游公司发展迅速, 同时, 电子信息一体化进程的加 快也使得移动智能终端迅速普及, 这为旅游业的发展提供了许多全新的交易经营模式。旅游 OTA (线上旅游平台) 模式对旅游业的供给和消费过程产生了深刻的影响, 使得企业的组织结 构发生了根本性的改变, 革新了人们旅游消费的观念, 发展前景十分广阔。

关键词：同程旅游网, OTA 模式, 旅游业

\section{1.引言}

面对广阔的发展机遇，我们同时不得不意识到旅游业是一个极具有竞争性的行业，尤其 在加入世贸组织后, 这种竞争便更具有国际化特征。因此, 虽然我国旅游业在短短几十年内 取得了显著的发展, 但其所面临的各种挑战也是我们无法回避的。2014 年, 国内在线旅游市 场稳坐头牌宝座的携程首次跌至亏损。同年, 途牛、无哪儿网也全部被迫加入亏损行列, 艺 龙亏损额度甚至相比去年进一步放大。因此, 在这样的市场形势下, 如何能分到旅游市场的 一杯美，并长期立于不败之地显然并不是一件容易的事!

作为近十几年发展起来的新兴产业, 旅游电子商务自身存在一定的先天不足, 如旅游企 业形象不突出, 效益不高, 网站内容简单信息量单一等。同程旅游网站多以搜索和信息发布 为主, 虽有部分涉及旅游线路的安排, 但大多数还是以订票, 订房为主, 少有自助游安排, 
旅游贴示等服务, 因而无法很好地完成客户个性化旅游的需要, 没有体现出 OTA 运营模式的 优势。此外, 全球经济尚未进入一个平衡稳定状态, 还有一个重要前提即线上支付手段还没 有体现出足够的普及率。国外 OTA 的迅速发展离不开全国范围内信用卡和网络服务的普及, 而国内线上支付手段 (如支付宝, 微信等) 尚未被大众所接受, 信用卡使用也存在一定的危 机漏洞, 这些因素无疑严重阻碍了国内旅游业 OTA 的发展 ${ }^{[1]}$ 。

在行业普遍亏损的情况下，同程旅游的成功并不是一个偶然的现象，它是这个创业团队 多年坚持的缩影。走出 “桃花源”, 从安逸到狼性, 只需要一场 “战争”。那么, 同程公司究竟 是怎样在这场战役中立于不败之地的? 在 OTA 发展的过程中, 同程企业又存在哪些问题? 。

\section{2 公司运营情况概述}

\section{1 公司及业务范围}

同程旅游（原称同程网）于 2004 年在苏州成立，是一个同时拥有 B2B, B2C 两大平台的旅 游电子商务网站, 通过十多年的在线市场的运行, 现已成为国内首屈一指的旅游电子商务平 台之一。十多年来, 公司斩获了大量的国内外荣誉。在线旅游市场 BATX 的派系之争中, 同程 是唯一一家获得携程, 腾迅两家投资的, 足以显得其纵横联合的能力非一般 ${ }^{[2]}$ 。2016 年 6 月, 同程旅游开始涉足另一大业务板块即同程旅游 OTA 平台, 主要是为了实现火车票、机票、酒 店等品牌业务的规模化盈利。

同程旅游业务涉及面广, 涵盖与旅游相关的方方面面，如机票，景点门票，酒店预订， 旅游软件开发, 旅游目的地攻略等。同时, 形成了以旅游攻略, 信息咨询等为特色的旅游社 区，被国内外媒体一致认为是旅游业中的“沃尔玛”。

\section{2 同程 OTA 的初体验}

OTA 电子商务发展有三大前提：（1）现代化和网络化的基础电子设施;（2）具备信息加 密, 安全认证, 电子支付, 物流服务, 售后服务保障等一系列政策, 法律环境; (3) 经济链条 的形成。与实体经济类似, OTA 运行必须存在相应的需求供应链, 主要指供方, 需方, 中间服 务供应商三大类型 ${ }^{[3]}$ 。

基于此，同程公司 OTA 管理问题主要体现在两个方面：外部宏观环境和内部管理机制。 同程公司 OTA 发展的宏观条件薄弱, 缺乏政府积极主导。事实上, 除了宏观环境外, 同程公 司内部的 OTA 管理机制也对运营产生了巨大的影响, 主要体现在战略管理（例如, 由于缺乏 创新机制带来的产品更新过慢)、员工职位培训、业务流程的标准化等, 这些都影响着同程公 司 OTA 的发展。所以, “修内功” 是同程公司当务之急。

\section{3 旅游公司 OTA 竞争格局}

在 2013 年, 携程准备涉足门票业务之前, 同程早和腾讯开展了一系列接洽活动, 并于 2014 年 2 月 14 日收到了来自腾讯等三家机构的投资, 资金高达五亿人名币。而携程原本计 划不通过控股参与竞争, 最终由于自身内部组织结构的变化, 以入股投资的方式结束了这场 门票之战, 成为了同程的第二大股东。虽然如此, 两家公司也在同程业务发展方面达成一致 意见, 携程表示两家公司仍然保持一种合作与竞争相结合的关系, 不会对同程具体业务进行 干涉 ${ }^{[4]}$ 。事实上, 同程应该对此次携程的投资表示感谢, 感谢的是携程的摚局行为, 因为在当 时几乎所有在线旅游行业都在烧钱, 不断亏损的状态下, 携程的加入让同程深刻体会到了危 机感, 并加入火热的竞争。也正因为如此, 同程 CEO 将 “再好别忘了危机和奋斗, 再难别忘 了梦想和坚持这两句话加入了公司的价值观当中”。

2012 年到 2013 年, 同程整体处于一种很舒适的状态, 因为在这段时间搜索引擎方面, 同 程一直都是做到了在线流量第一。2013 年下半年, 几乎所有的话题都与变得和移动互联网息 
息相关, 但此时 PC 端仍然是同程的主要战场, 移动端的收效微乎其微, 但当时运营仍处于还 不错的状态, 因此主动革命的情怀并没有被激发出来。但到 2014 年, 第一异位, 由携程和去 哪儿交替占领。同程的团队意识到, 眼看移动互联网这艘大船已经启航, 但遗憾的是同程目 前手里连一张 “船票” 也没有。同程团队想了很多办法来提高移动用户的所占比例, 通过一 系列的举措证明门票绑定是最有效的方式, 也就是挺有名的 “一元游” , 即同程包一个景点, 然后让游客低价进入, 比较成功的例如无锡的灵山大佛 ${ }^{[5]}$ 。这项营销活动成功地帮助同程搭上 了移动互联网这艘大船, 而这次的经历在 CEO 吴志祥看来更是团队的一次自我救赎。

成功进入线上旅游队伍的同程没有止步于门票业务, 同程选择以门票作为切入点, 延伸 到周边游以及国内游, 出境游等。而我们都知道, 途牛的主营业务正式出境游, 于是刚结束” 双程 “大战的同程发现竞争对手又由携程换成了途牛, 而这种竞争主要表现在广告等宣传公 关方面, 而公关宣传自然离不开砸广告, 疯狂烧钱等。与此同时, 行业也开始发生了很大的 变化, 随着大众点评与美团的业务联姻, 酒店预订成功被它们占领了大部分市场; 而携程通 过重重厮杀, 地位更加稳固, 因此, 在线旅游市场的格局似乎越来越清晰, 尽管同程有微信 这样巨大的流量入口，但整体的格局也是无法改变的。因此，公司 CEO 开始了一系列的市场 战略转型, 下面我们会具体介绍。

\section{4. 同程 OTA 现状}

\section{1 同程 OTA 结构特征}

同程旅游 OTA 模式主要包括三大结构: 企业管理信息系统, OTA 线上网站管理系统和 OTA 线上商城系统。OTA 的结构是企业运行 OTA 的主线, 企业按照结构设计原则指导企业各项事 务的完成 ${ }^{[6]}$ 。同程旅游 OTA 的结构特征具体表现为:

(1) 线上, 下信息互通互用

同程公司旅游 OTA 将 “电子商务” 和 “实体店铺” 相结合，优势互补。

（2）本地市场覆盖面广

对本地旅游市场的了解和熟悉是传统企业的最大优势, 同程公司 OTA 模式能够充分调动 企业已有资源, 加快与传统企业的优势互补进程, 实现商家, 网站, 本地消费者三者之间的 完美结合。

(3) 优化终端销售利益

线上平台的终端销售管理在企业中越来越重要, 企业根据授权掌握每一个线上平台, 线 上产品的实时销售数据, 并通过统一的机制达成订单的支付的管理, 统一进行资源的调度以 提高订单支付效率。另外, 线上平台改变了众多分店分别存储旅游产品, 设备的状况, 降低 企业成本的同时也优化了公司仓储结构。

(4) 有助于顾客忠诚度养成

通过网络技术能快速掌握顾客的消费反馈，基于此，企业可以提供更加个性化的服务以 获取更高黏度的高频率消费。在与消费者拉近距离的同时, 也提升了企业的市场价值和品牌 形象。

\section{2 同程 OTA 管理战略}

\subsection{1 外部战略}

同程公司 OTA 模式要想取得长远发展, 政府的规划指导必不可少, 比如旅游信息网的建 设, 信息系统发布, 宣传等都需要政府各方面的政策保障。此外, 政府部门工作还包括以下 几个方面: (1)促进 OTA 相关事业发展, 从事线上租赁设备, 维护系统等工作以维持中小旅游 企业的 OTA 进展; (2)完善旅游行业电子商务规范或进一步细化到 OTA 方面, 为线上, 线下的 消费提供法律方面的约束及保障; (3)加快与行业协会之间的合作，推动资源整合工作 ${ }^{[7]}$ 。

其次, 转变民众消费观念, 培养潜在客户群体, 具体就是消费者对公司线上平台的认识 
很大程度上影响着公司网络营销的进程。同程公司要拓展 OTA 业务则必须调动消费者线上消 费的积极性, 企业应与政府一道利用舆论的力量来更新大众的传统消费观念, 引导培养全新 的观念以推动 OTA 发展。另外, 移动支付时代来临, 必须多样化支付方式体验。OTA 支付之 战，不是基于技术，也不是基于标准，而是基于与消费者日常支付环节的契合程度。同程公 司应抓住支付节点的专业能力及与其他日常生活中的互动性, 使得这种开放式的支付方式成 为主流。

\subsection{2 内部战略}

公司在进行战略制定时必须将顾客的需求摆在首位，努力构建一个对顾客友好的线上营 销平台。在 OTA 推广过程中，同程公司重视顾客信任度的提高。同程公司服务周到，价位合 适, 除此之外还应注重一些安全隐患问题, 如网上支付的安全性, 顾客个人信息的保密性等。 在个性化旅游风靡的当今, 同程公司还尝试通过个性化服务满足顾客个性化的需求。OTA 坏 境为企业与顾客沟通建立了一个畅通的平台, 企业可以与顾客共同讨论旅游产品的设计和生 产。融有客户想法的产品有利于加强企业与顾客的粘性, 对长期保持信任关系具有重要的意 义。

虽然是线上交易平台，但完善的售后是产品营销的章节中极其重要的一部分。因为是旅 游产品, 而且线上完成交易看不到真实细致的存在, 所以交易效果可能有一定差异, 但正由 于如此，同程公司才更重视线下的售后服务，提高顾客消费满意度。同程公司在售后方面， 重视网站维护工作, 确保网站服务器运行稳定, 速度流畅, 杜绝消费者打开网站速度过慢, 网站瘫疾等现象发生 ${ }^{[8]}$ 。此外, 网站的专业设计不仅仅表现在外表美观, 还要突出的重中之重 就是顾客，通过设计让顾客体会到自己的受重视程度，查看内容舒适。

会员服务管理也是很重要的一个环节，与消费者的互动是与企业顾客友好型的体现，企 业主要按照以下操作：其一, 尽可能回答顾客提出的问题; 其二, 设置专为满足用户需求专 栏, 例如 ROM 家园; 其三, 建立网站的微博号, 并对外公布, 群的作用对于如火如茶的 OTA 发 展功不可没; 建立一个老顾客交流的社区互动系统, 顾客旅游后可以上传自己的旅行日志, 照片，心情等，并支付一定的登录积分。

在营销活动策划方面，公司定期举行企业营销专题活动，配合线上，线下的宣传以吸引 潜在顾客全体, 挽留老访客。如果奖励或礼品足够吸引顾客, 而顾客又很难获得时, 就会达 到一种病毒营销的效果。

\section{3 同程 OTA 市场竞争战略}

众所周知, 近两年国内旅游 OTA 市场一直处于混乱的竞争环境中, 不同与流量优先政策, 同程旅游意识到，用户体验才是影响消费者购买决策的关键。也正是因为有这样的认识，同 程旅游利用大数据为支撑, 启用用户与大数据相结合的战略模式, 力求通过本部外加其他六 个核心区域中心的辐射网络打造出新型的旅游发展模式。除了严抓业务量的同时, 同程还特 别关注服务质量的提升, 相关部门的数据表明, 关于旅游行业的相关投诉率, 同程旅游网是 最低的。

同程网某位高层表示, 就现阶段发展来看, 旅游行业正从标品竞争转向为非标品的竞争。 2016 年同程主要的目标是致力于非标品用户的积累，具体来说，同程希望重点投身于非标品 的海外游, 最好能把目前在标品中积累的消费者转移到非标品的海外游的业务中。值得注意 的是, 同程为了更好实现出境游战略, 还收购了三家具有出境游资格的旅行社, 采用与目的 地强者企业进行合作的战略, 增强自身的核心竞争力。仅 2015 年, 同程旅游就在周边的三个 国家成立了合资公司, 加快进行目的地市场的布局。同时他还表示, 同程在收效上的短期目 标是能够在两年后, 也就是 2018 年底, 营业收人达 500 亿元, 实现 10 亿元的利润收入, 同 时同程能够坐上线上旅游行业的首位宝座 ${ }^{[9]}$ 。

除了 “出发地” 和 “目的地” 两大市场战略以外，同程旅游还计划设立航空等相关产业 链的上下游公司来实现同程 OTA 闭环，进而提升用户体验。 


\section{5 同程 OTA 的特色化}

\section{1 聚焦海外游, 实现差异化}

因为战略调整的变化，2016 年被同程旅游定为公司的出境旅游年。为了配合公司的 “出境战略”，同程旅游推出一系列新的举措，例如除了公司本部苏州以外，另外在国内设立 四大运营中心作为出发地战略的重要组成, 在海外目的地开展日本旅游月活动、韩国定向观 光等, 都获得了用户较好的评价。

在不久的将来, 同程出境游还计划打造私人手机客服, 针对出境游的游客出现的问题实 现实时反馈, 争取实现 $0 \%$ 的投诉率; 另外针对运营过程中出现的中差评, 提升解决问题的效 率，不断完善自身的 CRM 系统。

\section{2 电商+脸商营销模式}

“电商+脸商”营销模式也就是我们在上面提到的“大数据+人”。不知道大家有没有发现, 在最新的同程旅游 APP 中多出了 “我的旅游顾问” 这一模块的内容，这一模块不仅可以及时 帮助你解决旅游中出现的问题，还会有一些旅游达人帮助你规划旅游行程等。这也是 “脸商” 模式的一种表现。除了达人经济外，明星不得不说是一种很好的 “脸商” 模式，在信息过剩 的今天，电视剧的营销方式有时候真的会让人眼前一亮，例如 2015 年的《克拉恋人》着实让 通灵珠宝火了一把。

因此，同程旅游开始注重自身的品牌宣传，前后与综艺节目以及旅游卫视等进行了 5 次 签约合作，同程旅游品牌公关经理表示，这一系列动作都意味着同程率先使用 “电商+脸商” 的运营模式，同程旅游借此成为中国第一注重发展自身旅游文化的旅行社。”

\section{3 涉足金融保险, 挖掘产品终端}

同程旅游涉足金融保险是公司旅游生态链的新动作，目的是为了弥补缺乏金融服务支持 的缺陷, 同时提升 OTA 整体生态链的活性。同程旅游推出的金融保险业务主要是针对产业链 的相关利益群体，例如为消费者和供应商提供资金等服务。此外，众筹项目也是同程旅游的 一个新的尝试, 主要推出一些有特色但不是很常见的产品。

还值得一提的就是同程最近还着眼于同程金融生态圈的打造，设立了包括跨境金融、小 微金融等在内的五大体系 ${ }^{[10]}$ 。

\section{6 同程 OTA 的展望}

随着互联网的风靡，每个公司都想分得旅游行业 OTA 的一杯美，为此，每个公司不断创 新，推出符合时代需求的营销模式。

途牛专注 “品牌+专线”，专线通常是升级版的服务，例如高于标准的餐饮和住宿，因人 而异安排消费者的行程等, 显然, 这样的模式无疑需要大量的人力和财力投入, 而且相应的 指标准则也应该更加完善。而同程选择了 “大数据+人”这种模式，选择这种模式的原因上面 已经提到了, 这里我们主要说下它存在的问题吧, 因为大数据和人的挖掘都离不开大量的人 力和财力，吸引消费者成本较高，所以，这种模式如果被其他公司采用后，那么又势必是一 场价格战，公司不得不付出更多的营销费用来吸引消费者的眼球 ${ }^{[11]}$ 。

每个公司业务主体存在一定差异，采用的营销战略也各有千秋，至于哪种战略是最符合 市场发展的，应该只有时间才是最具有说服力的。经历 OTA 发展的一波三折，同程未来 OTA 发展的路途也一定不会一帆风顺, 吴 CEO 还是要时刻保持清醒!

\section{参考文献}

[1] 魏善沛，电子商务网站开发与实现 [M]，北京，高等教育出版社，2014 年 
［2］方珺逸. 砸 60 亿，把它弄成中国最大旅行社，这是王健林的下一个 “小目标” ?

[EB/OL]. http://www. 360doc. com/content/16/0917/05/19476362_591385805. shtm1.

[3] 肖建成, 余蓉. 旅游电子商务在旅游业中的应用前景 $[J]$. 2014，（1）：275-275.

[4] 佚名. 在线旅游无战事, 梁建章卸任携程 CEO [EB/OL]. http://www. vccoo. com/v/a540f6.

[5] 张越. 依托网络优势促进中国旅游电子商务发展 $[J]$. 晋中学院学报. 2013, (4) : 104105.

[6] 王兆良. 我国旅游经济与电子商务相结合问题探讨 $[\mathrm{J}]$. 中南民族大学学报, $2012(3)$

[7] 杨丽. 中国旅游电子商务发展中的一些问题与对策的研究. 旅游学刊, 2011 (6)

[8] 熊晓辉. 同程旅游架构调整 拆分两大板块独立上市 [EB/OL]. http://www. wokeji. co.

[9］赵法涁. 同程旅游 2016 聚焦海外休闲游 [J]. 数字通信世界, - 2015(12)

[10］钟超. “科技+旅游” 同程旅游谋划两翼齐飞 [EB/OL]. http://tech. gmw. cn/2.

[11] 李冰. 在线旅游金融市场 2 年增 10 倍 5 大企业争相砸钱入场 $[\mathrm{N}]$. 证券日报, 2016-1$9(15)$ 